\title{
Approximation of Common Fixed Points of Two Strictly Pseudononspreading Multivalued Mappings in $\mathbb{R}$-Trees
}

\author{
Withun Phuengrattana \\ Department of Mathematics, Faculty of Science and Technology, Nakhon Pathom \\ Rajabhat University, Nakhon Pathom 73000, Thailand \\ e-mail : withun_ph@yahoo.com
}

Abstract. In this paper, we introduce and study a new multivalued mapping in $\mathbb{R}$ trees, called $k$-strictly pseudononspreading. We also introduce a new two-step iterative process for two $k$-strictly pseudononspreading multivalued mappings in $\mathbb{R}$-trees. Strong convergence theorems of the proposed iteration to a common fixed point of two $k$-strictly pseudononspreading multivalued mappings in $\mathbb{R}$-trees are established. Our results improve and extend the corresponding results existing in the literature.

\section{Introduction}

$\mathbb{R}$-trees were introduced by Tits [19] in 1977. Fixed point theory for singlevalued mappings in $\mathbb{R}$-trees was first studied by Kirk [10]. He proved that every continuous single-valued mappings defined on a geodesically bounded complete $\mathbb{R}$ tree always has a fixed point. Since then fixed point theorems for various types of single-valued and multivalued mappings in $\mathbb{R}$-trees has been rapidly developed and many of papers have appeared (see e.g. [1],[2],[3],[8],[12]). It is worth mentioning that fixed point theorems in $\mathbb{R}$-trees can be applied to graph theory, biology and computer science (see e.g., [4],[6],[11],[17]).

In 2009, Shahzad and Zegeye [18] proved strong convergence theorems of the Ishikawa iteration for quasi-nonexpansive multivalued mappings satisfying the endpoint condition in Banach spaces. Later in 2010, Puttasontiphot [15] obtained similar results in complete CAT(0) spaces. In 2012, Samanmit and Panyanak [16] introduced a condition on mappings in $\mathbb{R}$-trees which is more general than the endpoint condition, call it the gate condition, and proved strong convergence theorems of a modified Ishikawa iteration for quasi-nonexpansive multivalued mappings satisfying such condition.

Received December 26, 2013; accepted April 11, 2014.

2010 Mathematics Subject Classification: 47H09, 47H10.

Key words and phrases: fixed point, $\mathbb{R}$-tree, strictly pseudononspreading mapping, convergence theorems. 
In 2011, Osilike and Isiogugu [14] introduced a new single-valued mapping in a Hilbert space, namely $k$-strictly pseudononspreading. Recall that a single-valued mapping $T$ is called $k$-strictly pseudononspreading if

$$
\|T x-T y\|^{2} \leq\|x-y\|^{2}+k\|x-T x-(y-T y)\|^{2}+2\langle x-T x, y-T y\rangle
$$

for all $x, y \in D(T)$. In a Hilbert space, it is easy to show that (1.1) is equivalent to

$$
\begin{gathered}
(2-k)\|T x-T y\|^{2} \leq k\|x-y\|^{2}+(1-k)\|y-T x\|^{2}+(1-k)\|x-T y\|^{2} \\
+k\|x-T x\|^{2}+k\|y-T y\|^{2}
\end{gathered}
$$

for all $x, y \in D(T)$. Osilike and Isiogugu also proved weak and strong convergence theorems for approximating fixed points of $k$-strictly pseudononspreading mappings in Hilbert spaces.

However, up to now, no researchers have studied fixed point theorems for multivalued $k$-strictly pseudononspreading mappings even in Hilbert spaces, Banach spaces, CAT $(0)$ spaces and $\mathbb{R}$-trees setting. The propose of this paper is to introduce the concept of $k$-strictly pseudononspreading multivalued mappings and to obtain the strong convergence theorems for approximating a common fixed point of those multivalued mappings in the framework of $\mathbb{R}$-trees under the gate condition.

\section{Preliminaries}

Let $(X, d)$ be a metric space. A geodesic path joining $x \in X$ to $y \in X$ is a map $c$ from a closed interval $[0, l] \subset \mathbb{R}$ to $X$ such that $c(0)=x, c(l)=y$, and $d\left(c\left(t_{1}\right), c\left(t_{2}\right)\right)=\left|t_{1}-t_{2}\right|$ for all $t_{1}, t_{2} \in[0, l]$. In particular, $c$ is an isometry and $d(x, y)=l$. The image of $c$ is called a geodesic (or metric) segment joining $x$ and $y$. When it is unique this geodesic segment is denoted by $[x, y]$. For each $x, y \in X$ and $\alpha \in(0,1)$, we denote the point $z \in[x, y]$ such that $d(x, z)=\alpha d(x, y)$ by $(1-\alpha) x \oplus \alpha y$. The space $(X, d)$ is said to be a geodesic metric space if every two points of $X$ are joined by a geodesic, and $X$ is said to be uniquely geodesic if there is exactly one geodesic joining $x$ and $y$ for each $x, y \in X$.

A nonempty subset $D$ of $X$ is said to be convex if $D$ includes every geodesic segment joining any two of its points. A nonempty subset $D$ of $X$ is said to be gated if for any point $x \notin D$ there is a unique point $y_{x}$ such that for any $z \in D$,

$$
d(x, z)=d\left(x, y_{x}\right)+d\left(y_{x}, z\right) .
$$

Clearly gated sets in a complete geodesic space are always closed and convex. The point $y_{x}$ is called the gate of $x$ in $D$. It is easy to see that $y_{x}$ is also the unique nearest point of $x$ in $D$.

Definition 2.1. An $\mathbb{R}$-tree is a geodesic metric space $X$ satisfying

(i) there is a unique geodesic segment $[x, y]$ joining each pair of points $x, y \in X$;

(ii) if $[y, x] \cap[x, z]=\{x\}$, then $[y, x] \cup[x, z]=[y, z]$. 
It follows by (i) and (ii) that

(iii) if $u, v, w \in X$, then $[u, v] \cap[u, w]=[u, z]$ for some $z \in X$.

An $\mathbb{R}$-tree is a special case of a $\operatorname{CAT}(0)$ space. Note that a metric space $X$ is a complete $\mathbb{R}$-tree if and only if $X$ is hyperconvex with unique metric segments; see [9].

Let $T: D \rightarrow 2^{D}$ be a multivalued mapping. An element $z \in D$ is called fixed point of $T$ if $z \in T z$. An element $p \in D$ is said to be an endpoint of $T$ if $p$ is a fixed point of $T$ and $T p=\{p\}$ (see [20]). We shall denote by $F(T)$ the set of all fixed points of $T$ and by $\operatorname{End}(T)$ the set of all endpoints of $T$. It is easy to see that for each $T, \operatorname{End}(T) \subseteq F(T)$ and the converse is not true in general. If $\operatorname{End}(T)=F(T)$ then we say that $T$ satisfies the endpoint condition.

We shall denote the family of nonempty closed bounded subsets of $D$ by $C B(D)$, the family of nonempty closed convex subsets of $D$ by $C C(D)$, and the family of nonempty compact convex subsets of $D$ by $K C(D)$. The Hausdorff metric on $C B(D)$ is defined by

$$
H(A, B)=\max \left\{\sup _{x \in A} \operatorname{dist}(x, B), \sup _{y \in B} \operatorname{dist}(y, A)\right\} \text { for } A, B \in C B(D) .
$$

The multivalued mapping $T: D \rightarrow C B(D)$ is called

(i) nonexpansive if $H(T x, T y) \leq d(x, y)$ for all $x, y \in D$;

(ii) quasi-nonexpansive if $F(T) \neq \emptyset$ and $H(T x, T z) \leq d(x, z)$ for all $x \in D$ and $z \in F(T)$;

(iii) L-Lipschitzian if there exists a constant $L>0$ such that $H(T x, T y) \leq$ $L d(x, y)$ for all $x, y \in D$;

(iv) hemicompact if for any sequence $\left\{x_{n}\right\}$ in $D$ such that $\lim _{n \rightarrow \infty} \operatorname{dist}\left(x_{n}, T x_{n}\right)=$ 0 , there exists a subsequence $\left\{x_{n_{i}}\right\}$ of $\left\{x_{n}\right\}$ such that $\left\{x_{n_{i}}\right\}$ converges strongly to $p \in D$. We note that if $D$ is compact, then every multivalued mapping $T: D \rightarrow C B(D)$ is hemicompact.

It is clear that every nonexpansive multivalued mapping $T$ with $F(T) \neq \emptyset$ is quasi-nonexpansive but there exist quasi-nonexpansive mappings that are not nonexpansive.

Let $T: D \rightarrow C C(D)$ be a multivalued mapping with $F(T) \neq \emptyset$. We say that a point $u \in D$ is a key of $T$ if, for each $x \in F(T), x$ is the gate of $u$ in $T x$. We say that $T$ satisfies the gate condition if $T$ has a key in $D$. It is clear that the endpoint condition implies the gate condition but the converse is not true.

We now collect some basic properties of $\mathbb{R}$-trees.

Lemma 2.2. Let $X$ be a complete $\mathbb{R}$-tree. Then the following statements hold:

(i) [7] if $x, y, z \in X$ and $\alpha \in[0,1]$, then

$$
d(z, \alpha x \oplus(1-\alpha) y)^{2} \leq \alpha d(z, x)^{2}+(1-\alpha) d(z, y)^{2}-\alpha(1-\alpha) d(x, y)^{2} ;
$$


(ii) [7] if $x, y, z \in X$, then $d(x, z)+d(z, y)=d(x, y)$ if and only if $z \in[x, y]$;

(iii) [12] if $A$ and $B$ are bounded closed convex subsets of $X$, then

$$
d\left(P_{A}(u), P_{B}(u)\right) \leq H(A, B)
$$

for any $u \in X$, where $P_{A}(u), P_{B}(u)$ are respectively the unique nearest points of $u$ in $A$ and $B$;

(iv) [8] the gate subsets of $X$ are precisely its closed and convex subsets.

We state the following conditions in $\mathbb{R}$-trees:

A multivalued mapping $T: D \rightarrow C B(D)$ is said to satisfy condition (I) if there exists a nondecreasing function $f:[0, \infty) \rightarrow[0, \infty)$ with $f(0)=0$ and $f(r)>0$ for all $r>0$ such that $\operatorname{dist}(x, T x) \geq f(\operatorname{dist}(x, F(T)))$ for all $x \in D$.

Two multivalued mappings $T_{1}, T_{2}: D \rightarrow C B(D)$ are said to satisfy condition (II) if there exists a nondecreasing function $f:[0, \infty) \rightarrow[0, \infty)$ with $f(0)=0$ and $f(r)>0$ for all $r>0$ such that either $\operatorname{dist}\left(x, T_{1} x\right) \geq f\left(\operatorname{dist}\left(x, F\left(T_{1}\right) \cap F\left(T_{2}\right)\right)\right)$ or $\operatorname{dist}\left(x, T_{2} x\right) \geq f\left(\operatorname{dist}\left(x, F\left(T_{1}\right) \cap F\left(T_{2}\right)\right)\right)$ for all $x \in D$.

The following results are needed for proving our results.

Lemma 2.3.([13]) Let $X$ be a complete metric space, $A$ be a bounded closed subset of $X$, and $B$ be a compact subset of $X$. If $x \in A$ then there exists $y \in B$ such that $d(x, y) \leq H(A, B)$.

Proposition 2.4.([5]) Let $(X, d)$ be a complete metric space and $F$ be a nonempty closed subset of $X$. Let $\left\{x_{n}\right\}$ be a sequence in $X$ such that $d\left(x_{n+1}, p\right) \leq d\left(x_{n}, p\right)$ for all $p \in F$ and $n \in \mathbb{N}$. Then $\left\{x_{n}\right\}$ converges strongly to some point in $F$ if and only if $\lim _{n \rightarrow \infty} \operatorname{dist}\left(x_{n}, F\right)$.

\section{Main Results}

Definition 3.1. Let $D$ be a nonempty subset of a complete $\mathbb{R}$-tree $X$. A multivalued mapping $T: D \rightarrow C B(D)$ is called

(i) nonspreading if

$$
2 H(T x, T y)^{2} \leq \operatorname{dist}(y, T x)^{2}+\operatorname{dist}(x, T y)^{2}
$$

for all $x, y \in D$.

(ii) $k$-strictly pseudononspreading if there exists $k \in[0,1)$ such that

$$
\begin{aligned}
(2-k) H(T x, T y)^{2} \leq & k d(x, y)^{2}+(1-k) \operatorname{dist}(y, T x)^{2}+(1-k) \operatorname{dist}(x, T y)^{2} \\
& +k \operatorname{dist}(x, T x)^{2}+k \operatorname{dist}(y, T y)^{2}
\end{aligned}
$$

for all $x, y \in D$. 
Clearly every nonspreading multivalued mapping is 0-strictly pseudononspreading. It is clear that if $T$ is $k$-strictly pseudononspreading and has a fixed point, then for all $x \in D$ and $p \in F(T)$ we have

$$
H(T x, T p)^{2} \leq d(x, p)^{2}+k \operatorname{dist}(x, T x)^{2} .
$$

So, $T$ may not be quasi-nonexpansive. It is easy to show that if $T$ is a $k$-strictly pseudononspreading multivalued mapping with $F(T) \neq \emptyset$, then $F(T)$ is closed. Indeed, we let $\left\{x_{n}\right\}$ be a sequence in $F(T)$ such that $x_{n} \rightarrow x$ as $n \rightarrow \infty$. By the definition of $T$, we have

$$
\begin{aligned}
H\left(T x, T x_{n}\right)^{2} & \leq d\left(x, x_{n}\right)^{2}+k \operatorname{dist}(x, T x)^{2} \\
& \leq\left(d\left(x, x_{n}\right)+\sqrt{k} \operatorname{dist}(x, T x)\right)^{2} .
\end{aligned}
$$

Then

$$
H\left(T x, T x_{n}\right) \leq d\left(x, x_{n}\right)+\sqrt{k} \operatorname{dist}(x, T x) .
$$

It follows that

$$
\begin{aligned}
\operatorname{dist}(x, T x) & \leq \operatorname{dist}\left(x, T x_{n}\right)+H\left(T x_{n}, T x\right) \\
& \leq 2 d\left(x, x_{n}\right)+\sqrt{k} \operatorname{dist}(x, T x) .
\end{aligned}
$$

By letting $n \rightarrow \infty$ in above inequality, we have $(1-\sqrt{k}) \operatorname{dist}(x, T x) \leq 0$. Since $k \in[0,1)$, we get $\operatorname{dist}(x, T x)=0$. Hence, $x \in T x$ so that $F(T)$ is closed.

In order to prove our main results, the following lemma is needed.

Lemma 3.2. Let $D$ be a nonempty closed convex subset of a complete $\mathbb{R}$-tree $X$. Assume that $T: D \rightarrow K C(D)$ is a $k$-strictly pseudononspreading multivalued mapping. If $\left\{x_{n}\right\}$ is a sequence in $D$ such that $x_{n} \rightarrow x$ and $\operatorname{dist}\left(x_{n}, T x_{n}\right) \rightarrow 0$ as $n \rightarrow \infty$, then $x \in T x$.

Proof. Let $\left\{x_{n}\right\}$ be a sequence in $D$ such that $x_{n} \rightarrow x$ and $\operatorname{dist}\left(x_{n}, T x_{n}\right) \rightarrow 0$ as $n \rightarrow \infty$. For each $n$, let $y_{n} \in T x_{n}$ such that $d\left(x_{n}, y_{n}\right)=\operatorname{dist}\left(x_{n}, T x_{n}\right)$. Since $T x$ is compact, by Lemma 2.3 , there exist $z_{n} \in T x$ such that $d\left(y_{n}, z_{n}\right) \leq H\left(T x_{n}, T x\right)$. Then, we have

$$
\begin{aligned}
(2-k) d\left(y_{n}, z_{n}\right)^{2} \leq & (2-k) H\left(T x_{n}, T x\right)^{2} \\
\leq & k d\left(x_{n}, x\right)^{2}+(1-k) \operatorname{dist}\left(x, T x_{n}\right)^{2}+(1-k) \operatorname{dist}\left(x_{n}, T x\right)^{2} \\
& \quad+k \operatorname{dist}\left(x_{n}, T x_{n}\right)^{2}+k \operatorname{dist}(x, T x)^{2} \\
\leq & k d\left(x_{n}, x\right)^{2}+(1-k)\left(d\left(x, y_{n}\right)^{2}+d\left(x_{n}, z_{n}\right)^{2}\right)+k \operatorname{dist}\left(x_{n}, T x_{n}\right)^{2} \\
& \quad+k \operatorname{dist}(x, T x)^{2} \\
\leq & k d\left(x_{n}, x\right)^{2}+(1-k)\left(d\left(x, y_{n}\right)+d\left(x_{n}, z_{n}\right)\right)^{2}+k \operatorname{dist}\left(x_{n}, T x_{n}\right)^{2} \\
& \quad+k \operatorname{dist}(x, T x)^{2} \\
\leq & k d\left(x_{n}, x\right)^{2}+(1-k)\left(d\left(x, x_{n}\right)+2 d\left(x_{n}, y_{n}\right)+d\left(y_{n}, z_{n}\right)\right)^{2} \\
& +k \operatorname{dist}\left(x_{n}, T x_{n}\right)^{2}+k \operatorname{dist}(x, T x)^{2} .
\end{aligned}
$$


Compactness of Tx implies that there exists a subsequence $\left\{z_{n_{i}}\right\}$ of $\left\{z_{n}\right\}$ such that $z_{n_{i}} \rightarrow z \in T x$ as $i \rightarrow \infty$. Then, it follows by (3.1) that

$$
\lim _{i \rightarrow \infty} d\left(y_{n_{i}}, z_{n_{i}}\right) \leq \sqrt{k} \operatorname{dist}(x, T x) .
$$

This implies

$$
\begin{aligned}
d(x, z) & =\lim _{i \rightarrow \infty} d\left(x_{n_{i}}, z\right) \\
& \leq \lim _{i \rightarrow \infty} d\left(x_{n_{i}}, y_{n_{i}}\right)+d\left(y_{n_{i}}, z_{n_{i}}\right)+d\left(z_{n_{i}}, z\right) \\
& =\lim _{i \rightarrow \infty} d\left(y_{n_{i}}, z_{n_{i}}\right) \\
& \leq \sqrt{k} \operatorname{dist}(x, T x) \\
& \leq \sqrt{k} d(x, z) .
\end{aligned}
$$

Thus, $(1-\sqrt{k}) d(x, z)=0$. Since $k \in[0,1)$, it implies that $x=z \in T x$.

Theorem 3.3. Let $D$ be a nonempty closed convex subset of a complete $\mathbb{R}$-tree $X$. Let $T_{1}: D \rightarrow K C(D)$ be a $k$-strictly pseudononspreading multivalued mapping and $T_{2}: D \rightarrow K C(D)$ be a $k$-strictly pseudononspreading and L-Lipschitzian multivalued mapping with $\mathcal{F}=F\left(T_{1}\right) \cap F\left(T_{2}\right) \neq \emptyset$. Suppose that $T_{1}, T_{2}$ satisfy the gate condition. Let $u_{1}, u_{2}$ be keys of $T_{1}, T_{2}$, respectively. For $x_{1} \in D$, the sequence $\left\{x_{n}\right\}$ generated by

$$
y_{n}=\left(1-\alpha_{n}\right) x_{n} \oplus \alpha_{n} z_{n}^{(1)} \text { for all } n \in \mathbb{N},
$$

where $z_{n}^{(1)}$ is the gate of $u_{1}$ in $T_{1} x_{n}$, and

$$
x_{n+1}=\left(1-\beta_{n}\right) y_{n} \oplus \beta_{n} z_{n}^{(2)} \text { for all } n \in \mathbb{N},
$$

where $z_{n}^{(2)}$ is the gate of $u_{2}$ in $T_{2} y_{n}$. Let $\left\{\alpha_{n}\right\}$ and $\left\{\beta_{n}\right\}$ be sequences in $[0,1]$ such that $0<a \leq \alpha_{n}, \beta_{n} \leq b<1-k$. If one of the following is satisfied:

(i) $T_{1}, T_{2}$ satisfy condition (II),

(ii) $T_{1}$ or $T_{2}$ is hemicompact,

then $\left\{x_{n}\right\}$ converges strongly to an element of $\mathcal{F}$. 
Proof. For each $p \in \mathcal{F}$, we obtain by the gate condition and Lemma 2.2(i) that

$$
\begin{aligned}
d\left(x_{n+1}, p\right)^{2} \leq & \left(1-\beta_{n}\right) d\left(y_{n}, p\right)^{2}+\beta_{n} d\left(z_{n}^{(2)}, p\right)^{2}-\beta_{n}\left(1-\beta_{n}\right) d\left(y_{n}, z_{n}^{(2)}\right)^{2} \\
\leq & \left(1-\beta_{n}\right) d\left(y_{n}, p\right)^{2}+\beta_{n} d\left(P_{T_{2} y_{n}}\left(u_{2}\right), P_{T_{2} p}\left(u_{2}\right)\right)^{2}-\beta_{n}\left(1-\beta_{n}\right) d\left(y_{n}, z_{n}^{(2)}\right)^{2} \\
\leq & \left(1-\beta_{n}\right) d\left(y_{n}, p\right)^{2}+\alpha_{n} H\left(T_{2} y_{n}, T_{2} p\right)^{2}-\beta_{n}\left(1-\beta_{n}\right) d\left(y_{n}, z_{n}^{(2)}\right)^{2} \\
\leq & \left(1-\beta_{n}\right) d\left(y_{n}, p\right)^{2}+\beta_{n}\left(d\left(y_{n}, p\right)^{2}+k \operatorname{dist}\left(y_{n}, T_{2} y_{n}\right)^{2}\right) \\
& \quad-\beta_{n}\left(1-\beta_{n}\right) d\left(y_{n}, z_{n}^{(2)}\right)^{2} \\
= & d\left(y_{n}, p\right)^{2}-\beta_{n}\left(1-k-\beta_{n}\right) d\left(y_{n}, z_{n}^{(2)}\right)^{2} \\
\leq & \left(1-\alpha_{n}\right) d\left(x_{n}, p\right)^{2}+\alpha_{n} d\left(z_{n}^{(1)}, p\right)^{2}-\alpha_{n}\left(1-\alpha_{n}\right) d\left(x_{n}, z_{n}^{(1)}\right)^{2} \\
& \quad-\beta_{n}\left(1-k-\beta_{n}\right) d\left(y_{n}, z_{n}^{(2)}\right)^{2} \\
\leq & \left(1-\alpha_{n}\right) d\left(x_{n}, p\right)^{2}+\alpha_{n} d\left(P_{T_{1} x_{n}}\left(u_{1}\right), P_{T_{1} p}\left(u_{1}\right)\right)^{2}-\alpha_{n}\left(1-\alpha_{n}\right) d\left(x_{n}, z_{n}^{(1)}\right)^{2} \\
& \quad-\beta_{n}\left(1-k-\beta_{n}\right) d\left(y_{n}, z_{n}^{(2)}\right)^{2} \\
\leq & \left(1-\alpha_{n}\right) d\left(x_{n}, p\right)^{2}+\alpha_{n} H\left(T_{1} x_{n}, T_{1} p\right)^{2}-\alpha_{n}\left(1-\alpha_{n}\right) d\left(x_{n}, z_{n}^{(1)}\right)^{2} \\
& \quad-\beta_{n}\left(1-k-\beta_{n}\right) d\left(y_{n}, z_{n}^{(2)}\right)^{2} \\
\leq & \left(1-\alpha_{n}\right) d\left(x_{n}, p\right)^{2}+\alpha_{n}\left(d\left(x_{n}, p\right)^{2}+k \operatorname{dist}\left(x_{n}, T_{1} x_{n}\right)^{2}\right) \\
& \quad-\alpha_{n}\left(1-\alpha_{n}\right) d\left(x_{n}, z_{n}^{(1)}\right)^{2}-\beta_{n}\left(1-k-\beta_{n}\right) d\left(y_{n}, z_{n}^{(2)}\right)^{2} \\
= & d\left(x_{n}, p\right)^{2}-\alpha_{n}\left(1-k-\alpha_{n}\right) d\left(x_{n}, z_{n}^{(1)}\right)^{2}-\beta_{n}\left(1-k-\beta_{n}\right) d\left(y_{n}, z_{n}^{(2)}\right)^{2} . \\
& \quad
\end{aligned}
$$

This implies by $\alpha_{n}, \beta_{n}<1-k$ that $d\left(x_{n+1}, p\right) \leq d\left(x_{n}, p\right)$ for all $n \in \mathbb{N}$. Hence, $\left\{d\left(x_{n}, p\right)\right\}$ is nonincreasing and bounded below. It follows that $\lim _{n \rightarrow \infty} d\left(x_{n}, p\right)$ exists for each $p \in \mathcal{F}$. Using (3.2), we get that

$\alpha_{n}\left(1-k-\alpha_{n}\right) d\left(x_{n}, z_{n}^{(1)}\right)^{2}+\beta_{n}\left(1-k-\beta_{n}\right) d\left(y_{n}, z_{n}^{(2)}\right)^{2} \leq d\left(x_{n}, p\right)^{2}-d\left(x_{n+1}, p\right)^{2}$

This implies from $0<a \leq \alpha_{n}, \beta_{n} \leq b<1-k$ that

$$
\lim _{n \rightarrow \infty} d\left(x_{n}, z_{n}^{(1)}\right)=0 \text { and } \lim _{n \rightarrow \infty} d\left(y_{n}, z_{n}^{(2)}\right)=0 .
$$

Also

$$
\operatorname{dist}\left(x_{n}, T_{1} x_{n}\right) \leq d\left(x_{n}, z_{n}^{(1)}\right) \rightarrow 0 \text { as } n \rightarrow \infty .
$$


By the definition of $T_{2}$, we get that

$$
\begin{aligned}
\operatorname{dist}\left(x_{n}, T_{2} x_{n}\right) & \leq \operatorname{dist}\left(x_{n}, T_{2} y_{n}\right)+H\left(T_{2} y_{n}, T_{2} x_{n}\right) \\
& \leq d\left(x_{n}, z_{n}^{(2)}\right)+L d\left(y_{n}, x_{n}\right) \\
& \leq(1+L) d\left(x_{n}, y_{n}\right)+d\left(y_{n}, z_{n}^{(2)}\right) \\
& \leq(1+L)\left(d\left(x_{n}, z_{n}^{(1)}\right)+d\left(z_{n}^{(1)}, y_{n}\right)\right)+d\left(y_{n}, z_{n}^{(2)}\right) \\
& =(1+L)\left(d\left(x_{n}, z_{n}^{(1)}\right)+\left(1-\alpha_{n}\right) d\left(z_{n}^{(1)}, x_{n}\right)\right)+d\left(y_{n}, z_{n}^{(2)}\right) \\
& =(1+L)\left(2-\alpha_{n}\right) d\left(x_{n}, z_{n}^{(1)}\right)+d\left(y_{n}, z_{n}^{(2)}\right) \\
& \leq(1+L)(2-a) d\left(x_{n}, z_{n}^{(1)}\right)+d\left(y_{n}, z_{n}^{(2)}\right) .
\end{aligned}
$$

This implies by (3.3) that

$$
\operatorname{dist}\left(x_{n}, T_{2} x_{n}\right) \rightarrow 0 \text { as } n \rightarrow \infty .
$$

Case (i): $T_{1}, T_{2}$ satisfy the condition $(I I)$. Then, we have by (3.4) and (3.5) that $\lim _{n \rightarrow \infty} d\left(x_{n}, \mathcal{F}\right)=0$. By the closedness of $\mathcal{F}$ and Proposition 2.4, we have $\left\{x_{n}\right\}$ converges strongly to some point in $\mathcal{F}$.

Case (ii): $T_{1}$ or $T_{2}$ is hemicompact. Without loss of generality, we assume that $T_{1}$ is hemicompact. Then there exists a subsequence $\left\{x_{n_{i}}\right\}$ of $\left\{x_{n}\right\}$ such that $\left\{x_{n_{i}}\right\}$ converges strongly to $z \in D$. By (3.4) and (3.5), it follows by Lemma 3.2 that $z \in \mathcal{F}$. Since $\lim _{n \rightarrow \infty} d\left(x_{n}, p\right)$ exists for each $p \in \mathcal{F}$, it implies that $\left\{x_{n}\right\}$ converges strongly to $z \in \mathcal{F}$.

Since any nonspreading multivalued mapping is 0-strictly pseudononspreading multivalued mapping, the next corollary is obtained immediately from Theorem 3.3.

Corollary 3.4. Let $D$ be a nonempty closed convex subset of a complete $\mathbb{R}$ tree $X$. Let $T_{1}: D \rightarrow K C(D)$ be a nonspreading multivalued mapping and $T_{2}: D \rightarrow K C(D)$ be a nonspreading and L-Lipschitzian multivalued mapping with $\mathcal{F}=F\left(T_{1}\right) \cap F\left(T_{2}\right) \neq \emptyset$. Suppose that $T_{1}, T_{2}$ satisfy the gate condition. Let $u_{1}, u_{2}$ be keys of $T_{1}, T_{2}$, respectively. For $x_{1} \in D$, the sequence $\left\{x_{n}\right\}$ generated by

$$
y_{n}=\left(1-\alpha_{n}\right) x_{n} \oplus \alpha_{n} z_{n}^{(1)} \text { for all } n \in \mathbb{N},
$$

where $z_{n}^{(1)}$ is the gate of $u_{1}$ in $T_{1} x_{n}$, and

$$
x_{n+1}=\left(1-\beta_{n}\right) y_{n} \oplus \beta_{n} z_{n}^{(2)} \text { for all } n \in \mathbb{N},
$$

where $z_{n}^{(2)}$ is the gate of $u_{2}$ in $T_{2} y_{n}$. Let $\left\{\alpha_{n}\right\}$ and $\left\{\beta_{n}\right\}$ be sequences in $[0,1]$ such that $0<a \leq \alpha_{n}, \beta_{n} \leq b<1$. If one of the following is satisfied:

(i) $T_{1}, T_{2}$ satisfy condition (II),

(ii) $T_{1}$ or $T_{2}$ is hemicompact, 
then $\left\{x_{n}\right\}$ converges strongly to an element of $\mathcal{F}$.

Using the same arguments as in the proof of Theorem 3.3, we obtain the following result for approximating a fixed point of a $k$-strictly pseudononspreading multivalued mapping in $\mathbb{R}$-trees.

Theorem 3.5. Let $D$ be a nonempty closed convex subset of a complete $\mathbb{R}$-tree $X$. Let $T: D \rightarrow K C(D)$ be a $k$-strictly pseudononspreading multivalued mapping with $F(T) \neq \emptyset$. Suppose that $T$ satisfies the gate condition. Let $u$ be a key of $T$. For $x_{1} \in D$, the sequence $\left\{x_{n}\right\}$ generated by

$$
x_{n+1}=\left(1-\alpha_{n}\right) x_{n} \oplus \alpha_{n} z_{n} \text { for all } n \in \mathbb{N},
$$

where $z_{n}$ is the gate of $u$ in $T x_{n}$. Let $\left\{\alpha_{n}\right\}$ be a sequence in $[0,1]$ such that $0<a \leq$ $\alpha_{n} \leq b<1-k$. If either $T$ satisfy condition $(I)$ or $T$ is hemicompact, then $\left\{x_{n}\right\}$ converges strongly to an element of $F(T)$.

Proof. In Theorem 3.3, put $T_{1}=T$ and $T_{2}=I$ (identity mapping). Hence, we obtain the desired result from Theorem 3.3.

Remark 3.6. Theorem 3.3 extends [16] to the case of two $k$-strictly pseudononspreading multivalued mappings and our iteration process is different from the iteration process defined by Samanmit and Panyanak [16].

Acknowledgements. The author would like to thank the referees for valuable suggestions on the paper.

\section{References}

[1] A. G. Aksoy and M. A. Khamsi, A selection theorem in metric trees, Proc. Amer. Math. Soc., 134(2006), 2957-2966.

[2] S. M. A. Aleomraninejad, Sh. Rezapour and N. Shahzad, Some fixed point results on a metric space with a graph, Topology Appl., 159(2012), 659-663.

[3] A. Amini-Harandi and A. P. Farajzadeh, Best approximation, coincidence and fixed point theorems for set-valued maps in $\mathbb{R}$-trees, Nonlinear Anal., 71(2009), 1649-1653.

[4] I. Bartolini, P. Ciaccia and M. Patella, String matching with metric trees using an approximate distance, SPIR Lecture notes in Comput. Sci. 2476, Springer, Berlin, 1999.

[5] H. H. Bauschke and P. L. Combettes, Convex Analysis and Monotone Operator Theory in Hilbert Spaces, Springer, New York, NY, USA, 2011.

[6] M. Bestvina, $\mathbb{R}$-trees in topology, geometry, and group theory, in: Handbook of Geometric Topology, North-Holland, Amsterdam, 2002, pp. 55-91.

[7] S. Dhompongsa and B. Panyanak, On $\Delta$-convergence theorems in CAT(0) spaces, Comp. Math. Appl., 56(10)(2008), 2572-2579. 
[8] R. Espínola, W. A. Kirk, Fixed point theorems in $\mathbb{R}$-trees with applications to graph theory, Topology and its Applications, 153(2006), 1046-1055.

[9] W. A. Kirk, Hyperconvexity of $\mathbb{R}$-trees, Fundamenta Mathematicae, 156(1)(1998), 67-72.

[10] W. A. Kirk, Fixed point theorems in $C A T(0)$ spaces and $\mathbb{R}$-trees, Fixed Point Theory Appl., 2004(2004), 309-316.

[11] W. A. Kirk, Some recent results in metric fixed point theory, J. Fixed Point Theory Appl., 2(2007), 195-207.

[12] J. T. Markin, Fixed points, selections and best approximation for multivalued mappings in $\mathbb{R}$-trees, Nonlinear Anal., 67(2007), 2712-2716.

[13] S. B. Nadler Jr., Multi-valued contraction mappings, Pacific J. Math., 30(1969), 475488.

[14] M. O. Osilike and F. O. Isiogugu, Weak and strong convergence theorems for nonspreading-type mappings in Hilbert spaces, Nonlinear Anal., 74(2011), 1814-1822.

[15] T. Puttasontiphot, Mann and Ishikawa iteration schemes for multivalued mappings in $C A T(0)$ spaces, Applied Mathematical Sciences, 4(61)(2010), 3005-3018.

[16] K. Samanmit and B. Panyanak, On multivalued nonexpansive mappings in $\mathbb{R}$-trees, Journal of Applied Mathematics, Volume 2012, Article ID 629149, 13 pages.

[17] C. Semple and M. Steel, Phylogenetics, Oxford Lecture Ser. Math. Appl., vol. 24, Oxford Univ. Press, Oxford, 2003.

[18] N. Shahzad and H. Zegeye, On Mann and Ishikawa iteration schemes for multi-valued maps in Banach spaces, Nonlinear Anal., 71(2009), 838-844.

[19] J. Tits, A Theorem of LieKolchin for Trees. Contributions to Algebra: A Collection of Papers Dedicated to Ellis Kolchin, Academic Press, New York, 1977.

[20] K. Wlodarczyk, D. Klim and R. Plebaniak, Existence and uniqueness of endpoints of closed set-valued asymptotic contractions in metric spaces, J. Math. Anal. Appl., 328(2007), 46-57. 